\title{
A RETROSPECTIVE STUDY ON CONSTRICTIVE PERICARDITIS PATIENTS IN A TERTIARY CARE HOSPITAL
}

\section{Dr N Kavitha}

\section{Dr C Elavarasan*}

\section{MS, Mch, Assistant Professor Dept Of Cardiothoracic Surgery Tirunelveli Medical College, Tirunelveli}

MS,Mch Assistant Professordept Of Cardiothoracic Surgery Madras Medical College, Chennai * Corresponding Author

ABSTRACT Constrictive pericarditis is a syndrome characterised by thick, fibrotic and rigid pericardium caused by a wide spectrum of cardiac and non-cardiac conditions resulting in impaired diastolic filling with a high prevelance of morbidity and mortality. We sought to determine the etiology, age distribution, gender prediliction, symptomatology, surgical outcome and complications of constrictive pericarditis in our institute. A total of 11 patients who underwent pericardiectomy for constrictive pericarditis over a 3year period were studied retrospectively. The commonest etiology was idiopathic in $45.4 \%$ patients followed by tuberculosis in $27.3 \%$. Age distribution was between 17 years and 64 years. Males ( $81.2 \%$ ) were more affected than females. The Surgical approach was through left anterolateral thoracotomy in 4 patients and through median sternotomy in 7 patients. Cardiopulmonary bypass was used in 1 patient. Perioperative mortality was $18.2 \%$ causes being severe low cardiac output syndrome and bleeding. One year survival rate was $72.7 \%$.

\section{KEYWORDS : Constrictive Pericarditis, Pericardiectomy , Tuberculous Pericarditis.}

\section{BACKGROUND :}

Constrictive pericarditis (CP) usually results when the volume of the pericardial sac itself is sufficiently reduced relative to cardiac volume so that cardiac filling is impaired. The sac is almost always thickened [1]. In earlier decades in the west and in developing countries today, tuberculosis is the leading cause of constrictive pericarditis. [1] The etiology of chronic CP has changed over the past few decades, and TB is no longer the predominant cause $[3,4]$. The etiology of pericarditis is quite diverse and most patients progress to symptoms severe enough to require surgical intervention. Pericardiectomy is the treatment for pericardial constriction.. The risk factors associated with pericardiectomy have been reported, but the information is limited (especially for procedures conducted in India) [7]. The outcomes of pericardiectomy would greatly benefit from appropriate surgical strategies and perioperative medical management based on the identification of perioperative risk factors [6-9]. Here, we highlight the predictors of morbidity and mortality of pericardiectomy based on our experience of improving the management of CP. For this purpose, short-term surgical outcomes after pericardiectomy for $\mathrm{CP}$ were investigated in a single center.

\section{MATERIALS AND METHODS :}

We carried out a retrospective analysis of patients who underwent surgery for Constrictive pericarditis at Rajiv Gandhi Government General Hospital, Chennai. Between January 2014 and December 2016, we identified 11 patients diagnosed with $\mathrm{CP}$. The gender and age distribution are given by pictorial representation. Table 1 lists the causes of CP in this series.

\section{Fig 1. GENDER DISTRIBUTION}

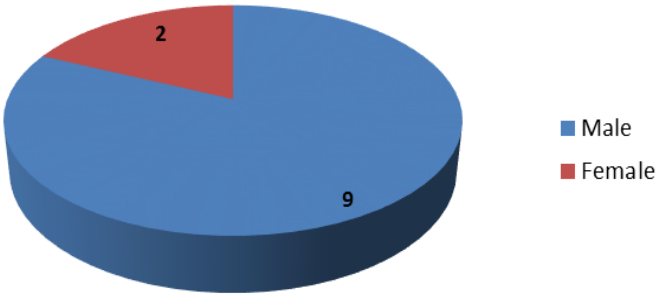

\section{Fig 2. AGE DISTRIBUTION}

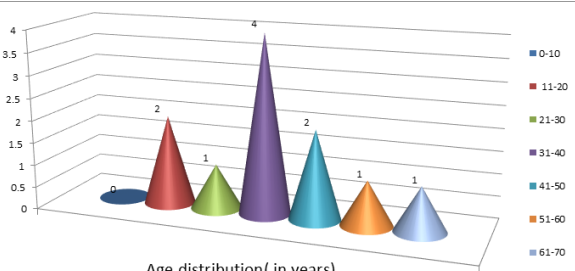

Age distribution( in years)

Table 1 :Relationship between constrictive pericarditis and the underlying etiology

\begin{tabular}{|l|l|l|}
\hline Etiologic factors & No & $\%$ \\
\hline Idiopathic & 5 & 64 \\
\hline Tuberculosis & 3 & 23 \\
\hline Bacterial infection & 2 & 1 \\
\hline Viral infection & 1 & 1 \\
\hline
\end{tabular}

The diagnosis of CP was made on the basis of clinical, echocardiography, computed tomography, or magnetic resonance imaging, surgical and pathological criteria. The most important diagnostic tool is the suspicion of $\mathrm{CP}$ in a patient with signs and symptoms of right-sided heart failure that are disproportionate to pulmonary of left-sided heart disease. Typical symptoms and signs are a prominent change in the $\mathrm{x}$ and $\mathrm{y}$ descent in jugular venous pulse, dyspnea upon exertion, palpitations, hepatomegaly, abdominal distension, as well as edema in the ankles or legs. Echocardiography revealed a severely thickened or calcified pericardium .

Between January 2014 and December 2016, 11 consecutive patients underwent pericardiectomy for CP. Surgical and pathological findings were reviewed to confirm the preoperative diagnosis.Among the 11 patients with $\mathrm{CP}, 2$ patients had a history of pulmonary Tuberculosis . Of which one had history of recurrent pericardial effusion for which she has undergone recurrent pericardiocentesis. Yet two another patients had similar history of recurrent pericardiocentesis , one with history of hepatitis B antigen positive and the other for pyopericardium. The median symptomatic duration before pericardiectomy was 11 months (range lmonth to 3 years ).

The baseline clinical details of the patients are summarized in Table 2. All 11 patients had dyspnea, 9 patients had diste 
nded jugular veins, 4 patients had hepatomegaly, 7 patients had pedal edema, 2 patients had ascitis, and 2 patients suffered from palpitations and chest pain. When classifying patients using the preoperative functional class set by the New York Heart Association (NYHA), 10 patients (90.9\%) were in class III/IV. Kussmaul's sign was seen in 3 patients. Two patients were icteric.

Initial laboratory assessments showed hypoalbuminemia in 5 patients, raised bilirubin in 2 . Elevated renal parameters noted in 4 patients. Chest radiography revealed pleural effusions ( $n=4)$, pulmonary infiltrates $(n=5)$, and pericardial calcification $(n=3)$. Electrocardiography revealed dimin ished QRS voltage in 9 patients , non-specific T-wave changes in 4 patients, atrial fibrillations in 2 patients. Echoca rdiogra phy revealed a thickened pericardium in 1 lpatients. Preop erative computed tomography (CT) or magnetic resonance imaging (MRI) was done in 10 patients, and part of the pericardium was demonstrated to be thickened or calcified.

Table 2. Descriptive data of clincial details

\begin{tabular}{|l|l|}
\hline VARIABLES & RESULTS \\
\hline NYHA functional class & \\
\hline II & $1(9.09 \%)$ \\
\hline III or IV & $7(63.6 \%)$ \\
\hline IV & $3(27.3 \%)$ \\
\hline Renal dysfunction & $1(1 \%)$ \\
\hline Cardiac symptom and signs & \\
\hline Dyspnea & $11(100 \%)$ \\
\hline Raised jugular vein distension & $9(81.8 \%)$ \\
\hline Pedal edema & $7(63.6 \%)$ \\
\hline Asscites & $2(18.2 \%)$ \\
\hline Palpitation & $2(18.2 \%)$ \\
\hline Hypoalbuminemia & $5(45.4 \%)$ \\
\hline Pleural effusion & $4(36.6 \%)$ \\
\hline Pericardial calcification & $5(45.4 \%)$ \\
\hline
\end{tabular}

${ }^{*} Q$ ualitative variables are presented as number(\%) and results of continuous variables as mean \pm standard deviation; Hypoalbuminemia defined as a serum albumin concentration of less than, was $21 \%$ at the time of admission in adult hospitalized patients.

\section{NYHA $=$ New York Heart Association}

All 11 patients underwent surgery, 7 patients through a median sternotomy, and through a left anterolateral thoracotomy in the remaining 4 patients. A relatively thin area of anterior pericardium was incised and a plane between the epicardium and the pericardium was established by sharp dissection. .Dissection was started at the base of the aorta, extended downwards to relieve the left ventricle first to prevent right ventricle distension. The anterior pericardium is freed $\mathrm{l} \mathrm{cm}$ anterior to left phrenic nerve to $l \mathrm{~cm}$ anterior to right phrenic nerve laterally, and from the anterior diaphragm inferiorly to the base of the great vessels superiorly. The pericardium over the right atrium and venae cavae was resected last. If dense epicardial scars were present, that areas were incised in a grid pattern . Cardiopulmonary bypass (CPB) was avoided during surgery, except for: one patientwho had right atrial tear while removing calcified spicules. In few cases we left small "islands" of calcified pericardial tissue which were so adherent.

For sternotomy a midline incision is made from just inferior to the xiphoid process. The entire extent of the sternum was divided in the midline. Left anterolateral thoracotomy through the fifth intercostal space was conducted in 4 patients. The decision for the approach was dependent on the personal preference of the operating surgeon. Left anterolateral thoracotomy offers excellent exposure of the anterolateral and inferior aspects of the left ventricle, with minimal manipulation and retraction of the left phrenic nerve. Carrying out dissection across the sternum and onto the right atrium or venae cavae during surgery is difficult. Hence, increasing number of surgeons prefer a midline incision and median sternotomy.

Surgical findings included a markedly thickened perica rdium, calcification (5/1), caseous necrosis in the pericardial cavity ( $1 / 11)$. The preoperative mean central venous pressure was $20.3 \pm 4.9 \mathrm{mmHg}$ and reduced to $8.8 \pm 3 . .2 \mathrm{mmHg}$ after surgery $(p<0.001)$. Surgical pathology specimens were examined for granulomas (3/11), as well as inflammation and fibrin deposition (6/11). Bacterial cultures was positive in one case.

Table 3 : Intra-operative and postoperative information for pericardiectomy

\begin{tabular}{|l|l|}
\hline Variable $^{*}$ & Result (\%) \\
\hline Intraoperative information: & \\
\hline Thoracotomy & $4(36.3 \%)$ \\
\hline Median sternotomy & $7(63.6 \%)$ \\
\hline Cardiopulmonary bypass & $1(9 \%)$ \\
\hline CVP change, mmHg & \\
\hline Preoperative CVP & $20.3 \pm 4.9$ \\
\hline Postoperative CVP & $8.8 \pm 3.2$ \\
\hline Postoperative complications & \\
\hline Low cardiac output syndrome & $5(45.45)$ \\
\hline Renal failure & $3(27.3)$ \\
\hline Respiratory insufficiency & $4(36.3)$ \\
\hline Hepatic dysfunction & $4(36.3)$ \\
\hline Intensive care unit stay, days & $3.5 \pm 1.7$ \\
\hline Hospital stay, days & $20 \pm 13.9$ \\
\hline In-hospital mortality & $2(18.2 \%)$ \\
\hline
\end{tabular}

${ }^{*}$ Qualitative variables are presented as number(\%) and results of continuous variables as mean \pm standard deviation.

$\mathrm{CVP}=$ central venous pressure.

Fig 3. CENTRAL VENOUS PRESSURE CHANGES (IN mm of $\mathrm{Hg}$ )

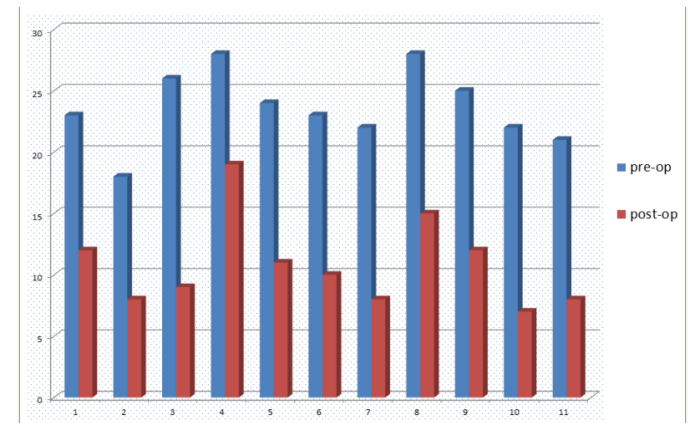

STATISTICAL ANALYSES

Results are the mean \pm standard deviation or percentages. For risk analyses, a multiple logistic regression model was developed using a forward stepwise variable selection method. The Analyses were undertaken using SPSS version 20.0 (SPSS, Chicago, IL,USA)

\section{RESULTS :}

All deaths were cardiac-related. They occurred in the perioperative period as a result of bleeding from right atrial rent while trying to release the calcified pericardium completely , cardio-pulmonary bypass was established thro ugh femoral route and repaired. But patient died in imm ed iate post operative period due to low cardiac output syndrome, 
massive blood transfusion. Another patient, of 64 years died on 18th post operative day due to low cardiac output syndrome, Respiratory insufficiency and renal failure. The $30-$ day mortality was $18.2 \%(2 / 11)$. Morbidity was due to lowcardiac-output syndrome $(n=5)$, acute renal failure $(n=3)$, respiratory insufficiency $(n=4)$ and hepatic dysfunction $(n=$ 4). All the discharged patients were followed up. Average follow up among patients was for a period of around 18 months ranging from 3 months to two and half years. A total of 7 patients had significant improvement in their NYHA status and 6 patients had a better outcome before discharge from hospital. After discharge from hospital, One patient died 3 months after surgery due to right heart failure. One-year survival in the operated patients was $72.7 \%$. Excluding the in hospital mortality, the one year survival in discharged patients was $88.9 \%$.

\section{DISCUSSION :}

The etiology of $\mathrm{CP}$ has varied between series during the past few decades. Idiopathic pericarditis is the predominant cause in the western world, TB was common cause of CP in "developing" and "underdeveloped" countries (especially in Asia) $[1,2,3]$. But now, Idiopathic pericarditis is increasingly common in developing countries like India, next to infection. Infection was either due Mycobacterium, staphylococcus or virus. Patients with radiation-induced heart disease (RIHD) were not observed in our study, and $18.2 \%$ patients had a history of lung TB. The mean age of 39 years in this series is about 10-years younger than that reported in earlier studies $[2,13]$. This finding reflects the difference in the etiology of CP.CP tends to be more common in males, and our study was consistent with previous studies [5,9]. There is no obvious explanation for this finding.

No single approach should be used to diagnose all cases of CP [3]. The diagnostic approach should be individualized for patients. The primary diagnosis varies, depending upon to whom patients were first referred. The prominent signs are pleural effusions, ascitis, pedal edema, and increased jugular venous pressure. Sometimes, however, patients may not present with sufficient signs or symptoms for a definitive diagnosis to be made. In such cases, additional imaging is required and recommended.

Echocardiography can provide important information for the diagnosis of $\mathrm{CP}$ and for its differentiation from restrictive cardiomyopathy, and should be the initial non-invasive imaging employed $[4,13]$. Compared with echocardiography, $\mathrm{CT}$ and MRI have proved good and reliable investigations that are available widely. They are recommended for patients whose symptoms and signs suggest the possibility of pericardial constriction. Minimal pericardial calcifications and fibrocalcific thickened layers are early detectable using CT / MRI chest . CT or MRI was carried out in all our patients and demonstrated calcification or thickening of the pericardium in 11 patients. Careful history-taking and physical examinations, as well as the appropriate use of echocardiography and high-resolution CT or MRI, may aid in an early diagnosis.[7]

Surgical management remains the only effective treatment for this potentially curable disorder $[6,10]$. In our experience, pericardiectomy should at least be considered if or before a patient develops NYHA functional class- III heart failure. In our center, pericardiectomy was carried out within $1 / 2$ weeks after the diagnosis. One case was taken as emergency who presented with shock. Various approaches and methods(left anterolateral thoracotomy; median sternotomy; $\mathrm{U}$ incision with the base of the U lying at the left sterna border; bilateral thoracotomy) have been described since Rehn and Sauerbruch conducted a successful pericardial resection for the chronic CP $[7,811]$. The most commonly used approach is median sternotomy (especially if pericardiectomy is carried out routinely using $\mathrm{CPB}$ ). The choice of approach appears is based on the personal preference of the surgeon.

Median sternotomy provides more radical clearance of the pericardium over the right atrium and venae cavae, and allows extensive pericardial removal if CPB is used[6,12]. In the present study, of all patients with pericardial thickening $\geq 3 \mathrm{~mm}, 4(36.3 \%)$ underwent left anterolateral thoracotomy, and7 (63.6\%) underwent median sternotomy. CPB aids in surgical dissection by emptying the ventricular cavities to define clearly the appropriate plane of dissection, and facilitates the management of inadvertent cardiac injury. In the event of inadvertent excessive bleeding $(n=1)$, CPB was established through femoral route as we opened that case through thoracotomy. The disadvantage of using CPB the potential for increased bleeding and other related complications. Chowdhury and colleagues suggested that delayed improvement and persistent symptoms are most commonly the result of incomplete decortications [9]. However, Schwefer et al suggested that long-term outcome is related not only to extent of the surgery but also the etiology of pericardial disease and preoperative NYHA status [12]. In the present study, despite complete release of pericardium 4patients had right heart failure post operatively

A correlation between NYHA class and overall or early survival has been observed by our research team and others scholars, and is the basis for advocating early pericard iectomy [11]. In this series, low cardiac output syndrome, respiratory insufficiency, hepatic dysfunction and renal failure were the most common postoperative complications. Improved perioperative management and medical therapy are important to avoid low cardiac output and restoration of right-heart function. Diuretics, inotropes, and vasodilators are the best medical therapies in the perioperative period [10]. However, there is a temptation, if faced with a much raised venous pressure and edema, for overzealous use of diuretics. Such management can lead to sudden death due to electromechanical dissociation.

In this series, the diagnosis of TB in CP was almost always confirmed by pathological means. Tuberculous CP can involve significant involvement of the lungs [2]. Preoperative chronic lung disease also has considerable negative effect on surgical results. Effective interventions can include aggressive physiotherapy, fiberoptic bronchoscopy along with aggressive screening for postoperative pneumonia. Blood transfusion may be needed for CP patients suffering from TB due to long-term malnutrition and deprivation of cardiogenic nutrients [7]. In general, 9-month anti-TB therapy for patients with suspected TB-based CP should be started after pericardiectomy.

\section{LIMITATION :}

Complete pericardiectomy may not achieve total restitution in some patients suffering from CP a long period before surgery. Long-term follow-up data on survival are lacking, which limits our ability to understand the long-term benefits of complete pericardiectomy. Radiation-induced pericarditis was has been reported to be a predictor of worse outcome, but we do not know if this was the cause in our patients. The only information regarding the etiology of $\mathrm{CP}$ in the present study was medical history and pathology. Our patients were done hemodynamic cardiac catheterization. Hence, there was increased difficulty in differentiating between constriction and restrictive cardiomyopathy.

\section{CONCLUSION}

Infection is the most common cause of constrictive pericarditis in our institution which is loaded with low socio economic 
groups. Idiopathic pericarditis is now increasing in devel oping countries like India and Tuberculous etiology is decreasing. Late presentation with advanced disease is more prevalent which makes the management difficult. Pericard iectomy was associated with a lower prevalence of mortality, and postoperative low cardiac output syndrome, as well as early normalization of hemodynamics. Careful perioperative management and surgical intervention upon or before rightheart failure may improve outcome. This is accomplished more readily through median sternotomy in patients with CP. Median sternotomy approach is preferred over thoracotomy since extensive dissection and CPB establishment in case of emergency is best through median sternotomy. Routine use of CPB during pericardiectomy is not necessary.One-year follow-up revealed that delayed presentation, New York Heart Association functional class III or IV, heavy pericardial calcification, poor pulmonary reserve and elevated liver function test were associated with increased mortality and morbidity.

\section{REFERENCES :}

1. Cameron J, Oesterle SN, Baldwin JC, Hancock EW. The etiologic spectrum of constrictive pericarditis. Am Heart J. 1987; 113:354 \pm 360 .

2. Schiavone WA. The changing etiology of constrictive pericarditis in a large referral center. Am J Cardiol. 1986;58:373 \pm 375

3. Deterling RA Jr, Humphreys GH II. Factors in the etiology of constrictive pericarditis. Circulation. 1955;12:30 \pm 43

4. Adebo OA, Adebonojo SA, Osinowo O, Falase AO, Grillo IA. Chronic constrictive pericarditis: hemodynamic changes following pericardiectomy. J Natl Med Assoc. 1980;72:461 \pm 466

5. Das PB, Gupta RP, Sukumar IP, Cherian G, John S. Pericardiectomy: indications and results. J Thorac Cardiovasc Surg. 1973;66:58 \pm 70

6. Levine HD. Myocardial fibrosis in constrictive pericarditis: electrocar diographic and pathologic observations. Circulation. 1973;48: 1268 \pm 1281

7. Seifert FC, Miller DC, Oesterle SN, Oyer PE, Stinson EB, Shumway NE. Surgical treatmentofconstrictive pericarditis: analysis of outcome and diagnostic error. Circulation. 1985;72(suppl II):II-264 \pm II-273

8. Potwar SA, Arsiwala SS, Bhosle KN, Mehta VI. Surgical treatment for chronic constrictive pericarditis. Indian Heart J. 1989;41:30 \pm 33 .

9. Bashi VV, John S, Ravikumar E, Jairaj PS, Shyamsunder K, Krishnaswami S. Early and late results of pericardiectomy in 118 cases of constrictive pericarditis. Thorax. 1988:43:637+641 1524

10. Wychulis AR, Connolly DC, McGoon DC. Surgical treatment of pericarditis. J Thorac Cardiovasc Surg. 1971;62:608 \pm 617

11. McCaughan BC, Schaff HV, Piehler JM, Danielson GK, Orszulak TA, Puga FJ, Pluth JR, Connolly DC, McGoon DC. Early and late results of pericardiectomy for constrictive pericarditis. J Thorac Cardiovasc Surg. 1985;89:340 \pm 350 .

12. Tirilomis T, Unverdorben S, von der Emde J. Pericardectomy for chronic constrictive pericarditis: risks and outcome. Eur J Cardiothorac Surg. $1994 ; 8: 487 \pm 492$.

13. Wood P. Chronic constrictive pericarditis. Am J Cardiol. 1961;7:48 \pm 61 